\title{
ON THE ENUMERATION OF MAGIC CUBES*
}

\author{
BY D. N. LEHMER
}

1. Introduction. Assume the cube with one corner at the origin and the three edges at that corner as axes of reference. In a magic cube the sum of the numbers in any line parallel to any one of the three axes is the same, and since the sum of all the numbers up to $N^{3}$ is $N^{3}\left(N^{3}+1\right) / 2$, the sum in each of the rows must be $N\left(N^{3}+1\right) / 2$. The square array in any plane parallel to a face of the cube forms a magic square according to the more general definition of a magic square as one in which the sum in each row and column is the same even if the numbers involved are other than the numbers from 1 to $N^{2}$ inclusive.

2. Transformations. We will call the square array in any plane parallel to a face of the cube a slab. It is clear that any permutation of a set of parallel slabs among themselves will not affect the magic property of a magic cube. There are manifestly $(N !)^{3}$ of these permutations, and by means of them we can bring any element into any desired cell of the cube. In particular we can bring the element $N^{3}$ to the origin, and after that we can, by a further permutation of the slabs, arrange the elements which lie on the three axes so that they read in descending order of magnitude from the origin out.

Again, by a rotation of the cube through an angle of $120^{\circ}$ about the diagonal through the origin, we may make a cyclic permutation of the three axes. We may then assume that the element next to the origin on the $x$ axis is larger than either of those next to the origin on the other two axes. Further, by a reflection of the whole cube in the $x y$ plane followed by a rotation about the $x$ axis of $90^{\circ}$, we may interchange the elements of the $y$ and $z$ axes without disturbing those on the $x$ axis. We may then assume that the element next to the origin on the $y$ axis is larger than that next to the origin on the $z$ axis. We then define a normal cube as one which has the element $N^{3}$ at the origin with the elements on the three axes arranged in descending order of magnitude from the origin out, and also such

* Presented to the Society, June 20, 1934. 
that the elements next to the origin are in descending order of magnitude reading from the $x$ to the $y$ to the $z$ axis in order. There is one and only one normalized cube obtainable from a given cube by the above $2 \cdot 3 \cdot(N !)^{3}$ transformations. The total number of different magic cubes of order $N$ is therefore equal to the number of normalized cubes of that order multiplied by $6(N !)^{3}$.

3. Magic Cubes of Order Three. There are no cubes of order 2. The magic sum would be 9 and, the largest element being 8 , only the element 1 could lie in the same row with it. For the magic cube of order 3 the magic sum is 42 and the other elements on the axes beside the element 27 must add to 15 . There are 7 pairs of elements available for the axes: $(14,1)(13,2)(12,3)(11,4)$ $(10,5)(9,6)(8,7)$. From these we may obtain 35 different types of cubes as follows:

$\begin{array}{lll}x \text { axis } & y \text { axis } & z \text { axis } \\ (14,1) & (13,2) & (12,3),(11,4),(10,5),(9,6)(8,7) \\ (14,1) & (12,3) & (11,4),(10,5),(9,6),(8,7) \\ (14,1) & (11,4) & (10,5),(9,6),(8,7) \\ (14,1) & (10,5) & (9,6),(8,7) \\ (14,1) & (9,6) & (8,7) \\ (13,2) & (12,3) & (11,4),(10,5),(9,6),(8,7) \\ (13,2) & (11,4) & (10,5),(9,6),(8,7) \\ (13,2) & (10,5) & (9,6),(8,7) \\ (13,2) & (9,6) & (8,7) \\ (12,3) & (11,4) & (10,5),(9,6),(8,7) \\ (12,3) & (10,5) & (9,6),(8,7) \\ (12,3) & (9,6) & (8,7) \\ (11,4) & (10,5) & (9,6),(8,7) \\ (11,4) & (9,6) & (8,7) \\ (10,5) & (9,6) & (8,7)\end{array}$

To exhibit the method of examining each of these 35 cases for possible magic cubes we give in detail the examination for the second case where the entries along the three axes are in order: $(14,1),(13,2)$ and $(11,4)$. We first list the possible slabs in the $x y$ plane which have for their top line the entries $27,14,1$ 
and for the left-hand column the entries $27,13,2$. These are got by assigning successive available elements for the center element, the others being then determined. None of these center elements must occur on the three axes, and none must give equal elements in the slab. The first condition bars out the numbers $1,2,4,11,13,14$ and 27 . Besides this the number 8 can not be used as that would give two bottom elements equal to 20. We get thus the following for the $x y$ plane:

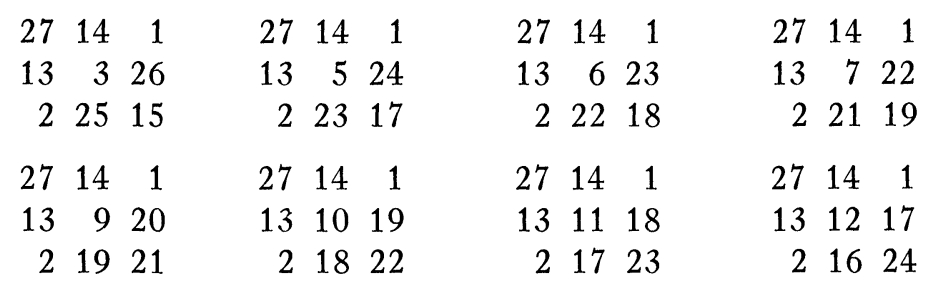

Similarly we list the possible slabs in the $y z$ plane. They are:

$\begin{array}{rrrrrrrrrrrrrrr}27 & 13 & 2 & 27 & 13 & 2 & 27 & 13 & 2 & 27 & 13 & 2 & 27 & 13 & 2 \\ 11 & 6 & 25 & 11 & 7 & 24 & 11 & 8 & 23 & 11 & 9 & 22 & 11 & 12 & 19 \\ 4 & 23 & 15 & 4 & 22 & 16 & 4 & 21 & 17 & 4 & 20 & 18 & 4 & 17 & 21\end{array}$

For the $z x$ plane we also have the following:

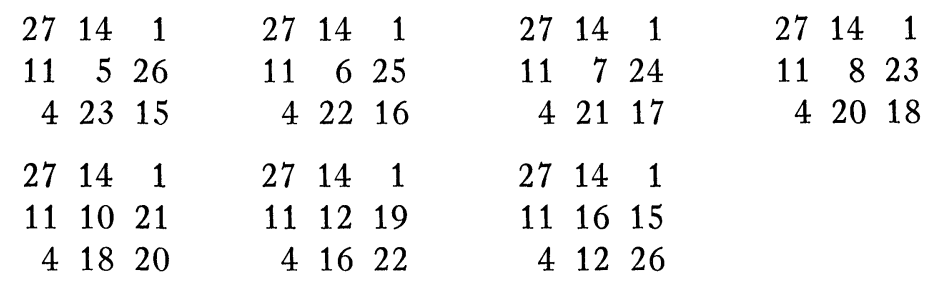

We select now a slab from the $x y$ plane, and a slab from the $y z$ plane and a slab from the $z x$ plane, taking care that none of the entries (except, of course, those that may lie on the axes) be repeated in any two slabs. Starting with the $x y$ slabs in order, the first set that appears is

\begin{tabular}{|c|c|c|}
\hline $\begin{array}{lll}27 & 14 & 1\end{array}$ & 271 & \\
\hline 13326 & 11 & 2 \\
\hline 22515 & 42 & 1 \\
\hline
\end{tabular}

Given these three slabs and the center of the cube, the rest of the elements of the cube are determined. This center element 
must again be chosen from the elements not already found in the three slabs. Since it lies in line with the three central elements of the slabs it is further restricted since the sum of the elements in those lines must be 42 . Now the only pairs which with 3 will give 42 are $(26,13),(25,14),(24,15),(23,16),(22,17),(21,18)$ and $(20,19)$, and neither number in any pair must have been used before. But this condition rules out every one of the pairs, and so there can be no cube with the above set of slabs in the three planes of reference.

The other sets of three slabs are treated similarly and all are ruled out at once except the set:

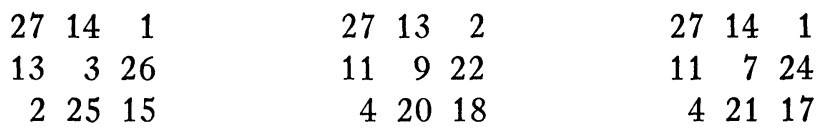

Here the central number 3 rules out all the available pairs except $(23,16)$, neither of which numbers appear in any of the slabs. When we try these numbers on the central element 7 , they are still both usable, since 7 and 23 demand 12 and 7 and 16 demand 19 and both of these numbers are still available. But the central element 9 of the third slab rules out the entry 16, since 9 and 16 demand 17 which is in the third slab already. But the number 23 is still left since 9 and 23 determine 10 which is still unused.

We proceed to derive then all the other elements of the cube, given the above three slabs and the centra! element 23. The result is our first magic cube. We give the three horizontal slabs in order from the bottom up:

\begin{tabular}{|c|c|c|c|c|}
\hline & $27 \quad 14 \quad 1$ & 11 & 24 & $\begin{array}{lll}421 & 17\end{array}$ \\
\hline (1) & 13326 & 92 & 10 & $2016 \quad 6$ \\
\hline & 22515 & 221 & 8 & $\begin{array}{ll}5 & 19\end{array}$ \\
\hline
\end{tabular}

In the same way all of the other cases were examined. Naturally certain short cuts appeared to abridge the work. The examinations were made three times, and in the third the cubes were normalized by putting the element 1 in the corner of the cube and arranging the elements on the three axes in ascending order out from the origin. Three and only three other cubes were obtained. The others are: 
$\begin{array}{lll}27 & 14 & 1\end{array}$

(2) $13 \quad 3 \quad 26$

22515

$27 \quad 14 \quad 1$

(3) $11 \quad 724$

$\begin{array}{lll}4 & 21 & 17\end{array}$

$\begin{array}{lll}27 & 13 & 2\end{array}$

(4) $11 \quad 922$

42018 $\begin{array}{lll}10 & 9 & 23\end{array}$

$\begin{array}{lll}8 & 22 & 12\end{array}$

$\begin{array}{lll}24 & 11 \quad 7\end{array}$

$\begin{array}{lll}10 & 9 & 23\end{array}$

62016

$\begin{array}{lll}26 & 13 & 3\end{array}$

$\begin{array}{lll}10 & 8 & 24\end{array}$

$\begin{array}{lll}6 & 19 & 17\end{array}$

$\begin{array}{lll}26 & 15 \quad 1\end{array}$ $\begin{array}{lll}5 & 19 & 18\end{array}$

$\begin{array}{lll}21 & 17 & 4\end{array}$

$\begin{array}{lll}16 & 6 & 20\end{array}$

$\begin{array}{lll}5 & 19 & 18\end{array}$

$\begin{array}{lll}25 & 15 & 2\end{array}$

$12 \quad 8 \quad 22$

52116

$\begin{array}{lll}25 & 14 & 3\end{array}$

$\begin{array}{lll}12 & 7 & 23\end{array}$

Each of these normalized cubes represents a group of 1296, so that there are altogether 5,184 magic cubes of order 3 .

The University of California

\title{
EXTENSION OF RANGE OF FUNCTIONS*
}

\author{
BY E. J. McSHANE
}

A well known and important theorem of analysis states that a function $f(x)$ which is continuous on a bounded closed set $E$ can be extended to the entire space, preserving its continuity. Let us consider a metric space $S$ and a function $f(x)$ defined and possessing a property $P$ on a subset $E$ of $S$. We shall for the sake of brevity say that $f(x)$ can be extended to $S$ preserving property $P$, if there exists a function $\phi(x)$, defined and possessing property $P$ on all of $S$, which is equal to $f(x)$ for all $x$ on $E$. Our present object is to establish an easily proved theorem which both includes the classical theorem stated above, and also shows that functions satisfying a Lipschitz or Hölder condition on an arbitrary set $E$ can be extended to $S$ preserving the Lipschitz or Hölder condition. An advantage of the present procedure is that it yields an explicit formula for the extension. $\dagger$

* Presented to the Society, June 20, 1934.

$\dagger$ After this paper was submitted for publication, the author found that Hassler Whitney had already indicated a simple proof that a function continuous on a bounded closed set can be extended to be continuous on all space, the method of extension being almost identical with the present one. $(\mathrm{H}$. Whitney, Transactions of this Society, vol. 36 (1934), footnote on p. 63). 